\title{
Tagungsbericht: 18. Tagung der lateinamerikanischen Antarktishistoriker in San Esteban, Chile (5.-7.9.2018), Eindrücke aus europäischer Sicht
}

\author{
Cornelia Lüdecke ${ }^{1, \text { 饮 }}$ \\ ${ }^{1}$ Universität Hamburg, Hamburg, Deutschland \\ ఓpensioniert
}

Correspondence: Cornelia Lüdecke (c.luedecke@lrz.uni-muenchen.de)

Published: 21 May 2021

2018 suchten die Veranstalter des XVIII Encuentro de Historiadores Antárticos Latinoamericanos (EHAL, Tagung der lateinamerikanischen Antarktishistoriker) erstmals den Kontakt zu einem europäischen Vertreter der Antarktisgeschichte, um einen neuen Blick auf das Forschungsthema zu erhalten. So wurde ich als Leiterin der Expertengruppe Geschichte der Antarktisforschung des Scientific Committee on Antarctic Research eingeladen, an der Tagung teilzunehmen, die von 5. bis 7. September 2018 von der Foundation Valle Hermoso und dem Centro des Estudios Hemisféricos y Polares im Hotel Termas El Corazón, San Esteban (Chile) am Fuße der Anden veranstaltet wurde. Der Tagungsort war sehr gut ausgewählt, denn er war nicht nur schön gelegen, sondern bot neben der Unterkunft, den benötigten Tagungsräumen auch eine angenehme familiäre Atmosphäre.

Am ersten Abend trafen sich alle Tagungsteilnehmer, die aus Argentinien, Chile, Deutschland, Kolumbien und Uruguay angereist waren, zum gemeinsamen Abendessen, kennenlernen und wiedersehen. Die folgenden zwei Tage waren mit 37 Vorträgen in zehn Sitzungen (davon zwei Parallelveranstaltungen) angefüllt. Bis auf meinen englischsprachigen Vortrag ist auf diesen Tagungen die allgemeine Vortragssprache Spanisch. Am Ende jeder Sitzung konnten, soweit es die Zeit noch erlaubte, Fragen gestellt werden. Die übliche Vortragsdauer betrug nur 10 Minuten, was für die Darstellung historischer Sachverhalte eigentlich viel zu kurz ist. Besser ging es mit den 20 Minuten dauernden Übersichtsvorträgen. Offenbar stehen traditionellerweise für dieses Treffen nur drei Tage zur Verfügung (Mittwoch Anreise, Donnerstag und Freitag Vorträge, Freitagnachmittag Abreise). Auffallend war, dass viele Studenten aus den verschiedensten
Master- und Promotionsprogrammen die Gelegenheit bekamen, ihre Ergebnisse vorzutragen und diskutieren zu lassen.

Nach der Eröffnung behandelte die erste Sitzung Exploration, Unglücke und Kontroversen im Zirkumantarktischen Ozean. Hier wurde anhand des überlieferten Logbuches das Schicksal der Brigg „Alabama Pocket“ in Antarktischen Gewässern, das Leben der Robbenschläger auf der Antarktischen Halbinsel und die Auswirkung der Expedition von Charles Wilkes auf die amerikanische Antarktisforschung bis zum 20. Jahrhundert dargestellt.Die zweite Sitzung thematisierte in drei Vorträgen aus chilenischer Sicht die politische Unsicherheit und Zugehörigkeit des Antarktischen Kontinents seit Beginn des 20. Jahrhunderts. Die dritte Sitzung widmete sich argentinischen, uruguayischen und chilenischen Perspektiven auf die Antarktis in der Mitte des 20. Jahrhunderts. In der vierten Sitzung wurde der gefrorene Kontinent als Ort der Wissenschaft herausgestellt und dabei auf die Veränderung der Meereisausdehnung, historische Aufzeichnungen der Discovery-Expedition sowie die moderne Kartographie mittels Drohnen eingegangen. Zum Abschluss der Vormittagssitzungen hielt Lydia Gómez den ersten Übersichtsvortrag über die argentinische Antarktishistoriographie unter dem Gesichtspunkt „Von der Idee zur Aktion: Bezüge, Reflexionen, Erfolge und Herausforderungen“".

Nach dem Mittagessen folgte die fünfte Sitzung über den antarktischen Kontinent zwischen Erstreben und Ersehen, in der eine Übersicht über die uruguayische Antarktisforschung, ein antarktischer Expeditionsplan von Coronel Hernán Pujato aus den 1950er Jahren, Gedanken zum Antarktisvertrag aus uruguayischer Sicht und die Entwicklung der antarktischen Geschichtskenntnisse in Argentinien vor- 


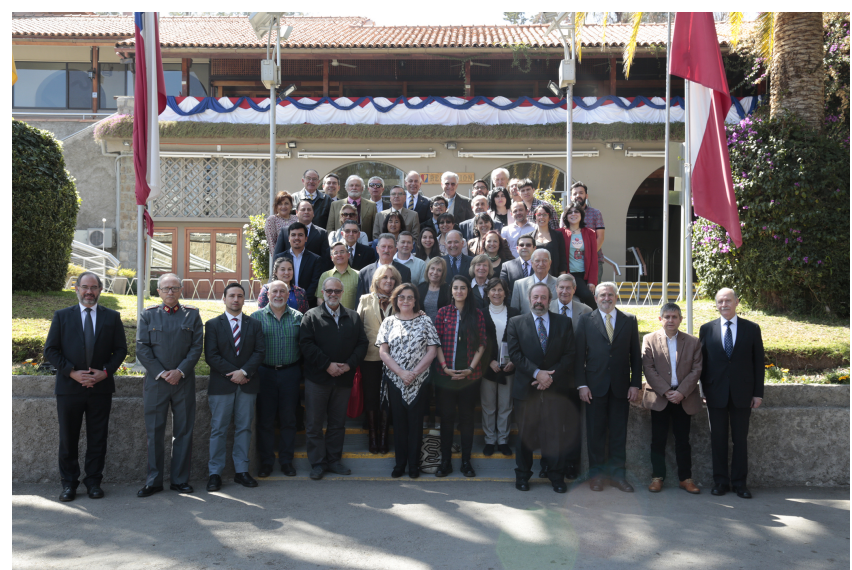

Abb. 1. Teilnehmer der 18. Tagung der lateinamerikanischen Antarktishistoriker in San Sebastian, Chile. (Foto: Valle Hermoso Foundation).

gestellt wurden. Parallel dazu fand die sechste Sitzung über den weißen Kontinent zwischen Alltäglichem und Außergewöhnlichem statt, in der das Maritime Museum in Ushuaia (argentinisches Feuerland), die Rolle der Mütter in der Antarktis und die Erinnerungen eines argentinischen Meteorologen auf der Antarktisstation San Martin an die damals verwendeten Schlittenhunde präsentiert wurden. Die siebte Sitzung beinhaltete Beiträge über Akteure, Hoffnungen und Kontroversen bezüglich der Antarktis, die drei wichtigen asiatischen Antarktismächte Japan, China und Südkorea in der Periode 1988 bis 2014 und die internationale Kontroverse über die Antarktis als Wasserreserve oder die Ausbeutung des Untergrundes in den Jahren 1990-2016. In der zeitgleichen achten Sitzung wurde das Wissen über die Antarktis besprochen, wie es beispielsweise im Lehrplan für Geschichte und Geographie hinsichtlich des von Chile beanspruchten antarktischen Territoriums oder mittels der Landkarten in argentinischen sozialwissenschaftlichen Lehrbüchern gelehrt wird. Auch wurde eine besondere Aktion vorgestellt, bei der Schulklassen nach vorheriger Einführung und Wissensvermittlung beispielsweise in Malwertbewerben zu antarktischen Themen ein spezielles „Antarktis-Siegel“ erwerben können. Die letzte Veranstaltung fand im Centro Cultural Pedro Aguirre Cerda in Calle Larga statt, das im einfachen chilenischen Landhaus des ehemaligen chilenischen Präsidenten untergebracht ist. Dort hielt Consuelo Léon Wöppke einen Vortrag über Pedro Aguirre Cerda und die Antarktis. Es folgten Wein, alkoholfreie Getränke und leckere Häppchen, bevor wir zum mehrgängigen Abendessen ins Hotel zurückfuhren.

Der Freitag war nicht ganz so mit Vorträgen überladen. Nachdem die Teilnehmer langsam eingetroffen waren, begann der Tag mit dem Übersichtsvortrag von Luis Valentín Ferrada über die Antarktis als Objekt der juristischen Regulation in Chile im Zeitraum 1892 bis 1918. Daran schlos-

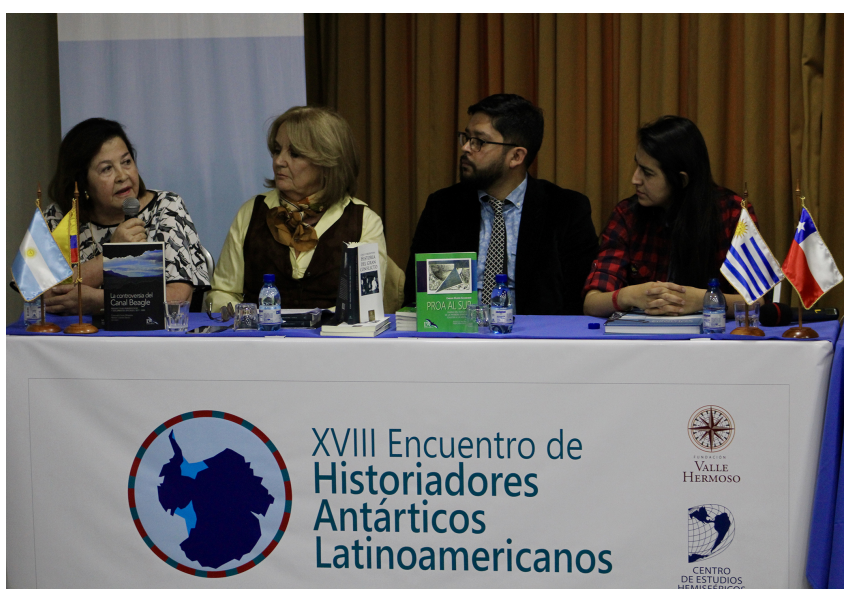

Abb. 2. V.l.n.r: Buchvorstellungen von Consuelo León Wöppke (Chile), Mónica Cerda (Chile), César Espinoza (Chile), und Natalia Jaramillo (Kolumbien). (Foto: Valle Hermoso Foundation).

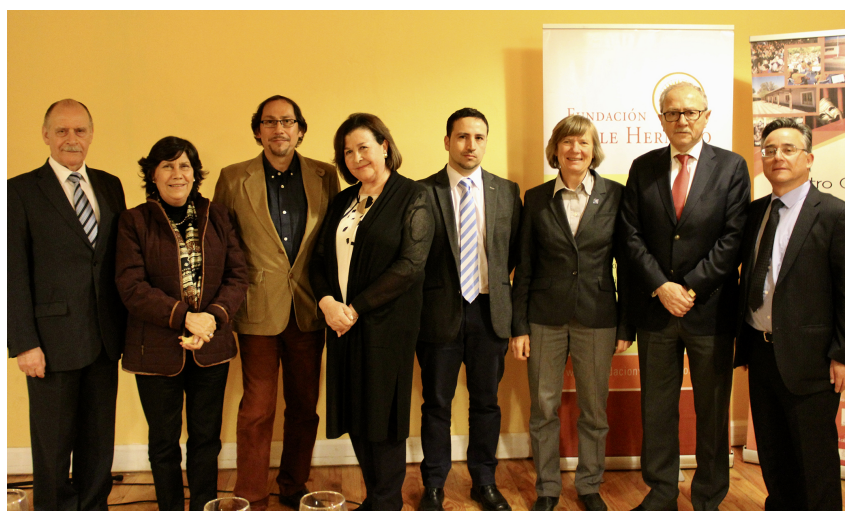

Abb. 3. V.l.n.r: Tagungsveranstalter und Gäste: Jorge Ilabaca (Valle Hermoso Stiftung), María Luisa Carvallo (Chilenisches Außenministerium), Edgardo Bravo (Kulturzentrum Pedro Aguirre Cerda), Consuelo León Wöppke (Valle Hermoso Stiftung), Nelson Llanos (Zentrum für Hemispherische und Polare Studien), Cornelia Lüdecke (Universität Hamburg), Eduardo Villalón (Chilenische Armee), and Mauricio Jara (Playa Ancha Universität). (Foto: Valle Hermoso Foundation).

sen sich wie auf diesen Tagungen üblich in der neunten Sitzung Buchvorstellungen an. Wir bekamen eine kurze Einführung in die ersten 25 Jahre der kolumbianischen Antarktisforschung, in das Tagebuch eines Teilnehmers an der ersten chilenischen Antarktisexpedition, in die Geschichte des „Großen Konfliktes“ zwischen Chile und Argentinien und in die Beagle Kanal Kontoverse aus der Sicht von Zeitungsartikeln und Dokumenten (1977-1985). Am Vormittag trafen weitere Teilnehmer ein, die anscheinend überwiegend aus dem Armebereich stammten und nun zu den Antarktisveteranen zählen. Ihr Interesse galt vor allem der zehnten Sitzung über die chilenische Antarktisstation Base O'Higgins (19481958). Hier trugen ehemalige Expeditionsteilnehmer (meist 


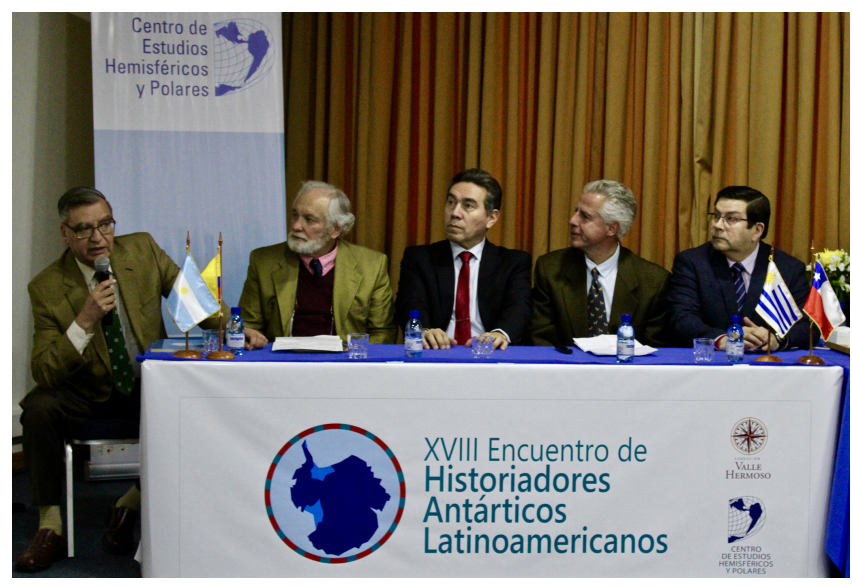

Abb. 4. V.l.n.r: Veteranen der chilenischen Antarktisstation Bernardo O'Higgins und das Jahr ihrer Überwinterungen: Humberto Julio (1968), Luis Komlos (1988), Leonardo Pérez 1978), Hugo Mahuzier (1992) and Eduardo Vilaza (2003-2009). (Foto: Valle Hermoso Foundation).

Offiziere) ihre Erinnerungen und Erfahrungen vor. Diese Art von Erinnerungskultur auf Tagungen erscheint uns fremd, ist aber offenbar ein fester Bestandteil dieser lateinamerikanischen Tagungen. Die Tagung endete mit meinem Vortrag über die Geschichte des deutschen Engagements in der Antarktis, der mit sehr großem Interesse aufgenommen wurde, weil darüber in Lateinamerika wenig bekannt ist. Nach dem gemeinsamen Mittagessen wurden die Teilnehmer mit einem Bus zum Flughafen nach Santiago gebracht.

Die Tagung verlief nicht so straff organisiert, wie wir es üblicherweise von westlich orientierten Tagungen gewohnt sind. Zum einen wird der im Programm ausgedruckte Tagesbeginn grundsätzlich um mehr als 15 Minuten überzogen, bis alle Teilnehmer langsam eingetrudelt sind. Auch die Vortragsdauer wurde oft nicht eingehalten, was bei nur 10 Minuten Redezeit zwar nachvollziehbar ist, aber den Gesamtablauf sehr stören kann. Selbst eine Ampel mit grüner, gelber und roter Lampe hat manche nicht davon abgehalten, die zur Verfügung stehende Zeit bei weitem zu überziehen. Dies sei allerdings eine typisch lateinamerikanische Verhaltensweise, wurde mir erklärt.
Neben Masterstudenten und Doktoranden haben Antarktishistoriker, Veteranen aus den Anfängen der lateinamerikanischen Antarktisforschung, Wissenschaftler, Lehrer, Politikwissenschaftler und Diplomaten an der Tagung teilgenommen. Es ist schon erstaunlich, dass EHAL jedes Jahr mit einer vergleichsweise großen Interessentenzahl durchgeführt werden kann und immer wieder neue Beiträge liefert. Leider gibt es außer einem Übersichtsprogramm für die Teilnehmer keine Zusammenfassungen der Vorträge.

Datenverfüg barkeit. Für diesen Artikel wurden keine Datensätze genutzt.

Interessenkonflikt. Die Autor*innen erklären, dass kein Interessenkonflikt besteht.

Begutachtung. This paper was edited by Bernhard Diekmann. 